\title{
QUEEN'S
UNIVERSITY
BELFAST
}

\section{Isotope Shift in the Dielectronic Recombination of Three-Electron}

ANd57+

Brandau, C., Kozhuharov, C., Harman, Z., Muller, A., Schippers, S., Kozhedub, Y. S., Bernhardt, D., Boehm, S., Jacobi, J., Schmidt, E. W., Mokler, P. H., Bosch, F., Kluge, H. J., Stohlker, T., Beckert, K., Beller, P., Nolden, F., Steck, M. Gusftqeridze, A., ... Stachura, Z. (2008). Isotope Shift in the Dielectronic Recombination of ThreeElectron ${ }^{\mathrm{Ad}}{ }^{2}$. Physical Review Letters, 100(7), [073201]. https://doi.org/10.1103/PhysRevLett.100.073201

\section{Published in:}

Physical Review Letters

Document Version:

Publisher's PDF, also known as Version of record

Queen's University Belfast - Research Portal:

Link to publication record in Queen's University Belfast Research Portal

Publisher rights

(C) 2008 The American Physical Society

\section{General rights}

Copyright for the publications made accessible via the Queen's University Belfast Research Portal is retained by the author(s) and / or other copyright owners and it is a condition of accessing these publications that users recognise and abide by the legal requirements associated with these rights.

Take down policy

The Research Portal is Queen's institutional repository that provides access to Queen's research output. Every effort has been made to ensure that content in the Research Portal does not infringe any person's rights, or applicable UK laws. If you discover content in the Research Portal that you believe breaches copyright or violates any law, please contact openaccess@qub.ac.uk. 


\title{
Isotope Shift in the Dielectronic Recombination of Three-Electron ${ }^{A} \mathrm{Nd}^{57+}$
}

\author{
C. Brandau, ${ }^{1, *}$ C. Kozhuharov, ${ }^{1}$ Z. Harman, ${ }^{2}$ A. Müller, ${ }^{3}$ S. Schippers, ${ }^{3}$ Y. S. Kozhedub, ${ }^{4}$ D. Bernhardt, ${ }^{3}$ S. Böhm, ${ }^{3}$ \\ J. Jacobi, ${ }^{3}$ E. W. Schmidt, ${ }^{3}$ P. H. Mokler, ${ }^{2,3}$ F. Bosch, ${ }^{1}$ H.-J. Kluge, ${ }^{1}$ Th. Stöhlker, ${ }^{1}$ K. Beckert, ${ }^{1}$ P. Beller, ${ }^{1}$ F. Nolden, ${ }^{1}$ \\ M. Steck, ${ }^{1}$ A. Gumberidze, ${ }^{1}$ R. Reuschll, ${ }^{1}$ U. Spillmann, ${ }^{1}$ F. J. Currell, ${ }^{5}$ I. I. Tupitsyn, ${ }^{4}$ V. M. Shabaev, ${ }^{4}$ U. D. Jentschura, ${ }^{2}$ \\ C. H. Keitel, ${ }^{2}$ A. Wolf, ${ }^{2}$ and Z. Stachura ${ }^{6}$ \\ ${ }^{1}$ Gesellschaft für Schwerionenforschung (GSI), 64291 Darmstadt, Germany \\ ${ }^{2}$ Max-Planck Institut für Kernphysik, 69117 Heidelberg, Germany \\ ${ }^{3}$ Institut für Atom-und Molekülphysik, Justus-Liebig-Universität, 35392 Giessen, Germany \\ ${ }^{4}$ Department of Physics, St. Petersburg State University, 198504 St. Petersburg, Russia \\ ${ }^{5}$ Physics Department, Queen's University, Belfast BT7 1NN, United Kingdom \\ ${ }^{6}$ Instytut Fizyki Jadrowej, 31-342 Kraków, Poland
}

(Received 28 September 2007; published 19 February 2008)

Isotope shifts in dielectronic recombination spectra were studied for Li-like ${ }^{A} \mathrm{Nd}^{57+}$ ions with $A=142$ and $A=150$. From the displacement of resonance positions energy shifts $\delta E^{142150}\left(2 s-2 p_{1 / 2}\right)=$ $40.2(3)(6) \mathrm{meV}[(\mathrm{stat})(\mathrm{sys})]$ and $\delta E^{142150}\left(2 s-2 p_{3 / 2}\right)=42.3(12)(20) \mathrm{meV}$ of $2 s-2 p_{j}$ transitions were deduced. An evaluation of these values within a full QED treatment yields a change in the meansquare charge radius of ${ }^{142}{ }^{150} \delta\left\langle r^{2}\right\rangle=-1.36(1)(3) \mathrm{fm}^{2}$. The approach is conceptually new and combines the advantage of a simple atomic structure with high sensitivity to nuclear size.

DOI: 10.1103/PhysRevLett.100.073201

The extent and shape of the proton distribution are basic properties of the atomic nucleus and reflect the interplay of forces that act between the nuclear constituents, protons, and neutrons. The gross properties of nuclear charge radii across the chart of nuclides - and also the fine details within isotope or isotone chains - provide primary observables for testing nuclear models [1-3]. Charge radii, nuclear shape, and hyperfine structure studies with classical methods such as electron scattering and x-ray spectroscopy of electronic and muonic atoms are essentially restricted to stable nuclei. Thus those isotopes far off the valley of stability cannot be addressed where the predictive power of nuclear models is poor, and interesting phenomena, like neutron or proton skins and shape effects occur. This limitation is overcome in optical isotope shift (OIS) measurements which are well suited to study long isotopic chains including exotic nuclides [4]. OIS is highly sensitive and possesses an enormous experimental precision. Since measurements of OIS are usually limited to low charge states, the interpretation of the data is, however, hampered by atomic many-body effects. The difficulty of a reliable theoretical description of these complex electronic configurations results in large uncertainties due to specific mass shift contributions and in the calculation of the change of the electronic density at the site of the nucleus.

Detailed knowledge about nuclear properties and their influence on electron binding is essential for many applications in modern atomic physics research, in quantum chemistry [5], and even in the determination of chemical abundances of stellar objects [6]. This is particularly true for high-precision tests of fundamental interactions like atomic parity nonconservation [7-9] or quantum electrodynamics (QED) in strong electromagnetic fields [10-12].
PACS numbers: $34.80 . L x, 21.10 . F t, 31.30 . G s, 31.30 . J-$

Nuclear effects play a critical role in such tests and limit their explanatory power. The constraints are increasingly more severe for heavier elements since the scaling of nuclear effects with the nuclear charge $Z$ exceeds the one of the sought-after contributions [13,14].

Radii compilations such as $[4,15,16]$ are widely used for reference. A closer look into the publications that form the basis of the compilations very often reveals inconsistencies. For instance, for the present case of neodymium, more than 20 publications from all four classical techniques can be found (for references, see $[4,15,16])$. In [17] a decreasing mean-square charge radius for a change from isotope ${ }^{142} \mathrm{Nd}$ to ${ }^{150} \mathrm{Nd}$ is reported, whereas in [18] a radius increase and a value of only ${ }^{142150} \delta\left\langle r^{2}\right\rangle=-0.22 \mathrm{fm}^{2}$ are reported. While these values are considered as outliers the bulk of the data still covers a range from about ${ }^{142}{ }^{150} \delta\left\langle r^{2}\right\rangle=-1.20 \mathrm{fm}^{2}$ to $-1.36 \mathrm{fm}^{2}$.

In view of the wide range of applications and the scatter of the experimental data alternative methods for a reliable determination of charge radii are highly desirable. In this Letter we present a conceptually different experimental access to charge radii changes that is particularly well suited for the investigation of heavy stable and also unstable nuclides. The method is based on the storage ring measurement of isotope shifts (IS) $\delta E^{A, A^{\prime}}$ in the spectrum of resonant electron-ion recombination of heavy fewelectron ions. The technique is demonstrated for the case of the two stable even-even isotopes ${ }^{142} \mathrm{Nd}^{57+}$ and ${ }^{150} \mathrm{Nd}^{57+}$ of Li-like neodymium. Storage rings provide very clean experimental conditions since isotopically pure, quasimonoenergetic ("cooled") beams in a single charge state are available. The experiments were performed at the electron cooler of the heavy ion storage 
ring ESR [19] at GSI in Darmstadt, Germany. The benefit of using few-electron ions for IS studies was previously shown employing classical spectroscopy at electron-beam ion traps (e.g., $[20,21])$. Because of the simplicity of the atomic configurations, the interpretation of the data is clear and without ambiguity. For the $2 s \rightarrow 2 p$ transitions of Lilike ions the electronic part can be treated theoretically with high accuracy. Many-body and mass effects are small and can be reliably accounted for.

Resonant electron-ion recombination - also termed dielectronic recombination (DR) - can be viewed as a twostep process. The first step of DR is called dielectronic capture (DC) and is time-reverse to autoionization. Free electrons that possess matching kinetic energy can recombine resonantly and excite a bound electron. If -in a second step-DC is succeeded by emission of photons, DR is complete. For the $2 s \rightarrow 2 p_{j}(j=1 / 2,3 / 2)$ excitations of ${ }^{A} \mathrm{Nd}^{57+}$ DR can be described by

$$
\begin{aligned}
e^{-}+{ }^{A} \mathrm{Nd}^{57+}\left(1 s^{2} 2 s_{1 / 2}\right) & \rightarrow{ }^{A} \mathrm{Nd}^{56+}\left(1 s^{2} 2 p_{j} n l_{j^{\prime}}\right)^{* *} \\
& \rightarrow{ }^{A} \mathrm{Nd}^{56+}+\text { photons. }
\end{aligned}
$$

In $\mathrm{Nd}^{57+}$, DC can proceed via Rydberg states with principal quantum numbers $n \geq 18$ for the $2 s \rightarrow 2 p_{1 / 2}$ core excitations and $n \geq 8$ for $2 s \rightarrow 2 p_{3 / 2}$, respectively (compare Figs. 1 and 2). With increasing electron-ion collision energy, a series of Rydberg resonances up to the series limits $n \rightarrow \infty$ are formed [10,22]. For different isotopes, the whole resonance series exhibits the same energy shift, i.e., the one of the corresponding core excitation. The captured loosely bound Rydberg electron itself is almost unaffected by the small variations of the nuclear potential and its contribution to the isotope shift can be safely neglected.

In recent DR experiments at the storage rings CRYRING in Stockholm and TSR in Heidelberg, the high resolution that can be achieved for exceptionally low-energy reso-



FIG. 1 (color online). Dielectronic recombination of the Lilike neodymium isotopes ${ }^{142} \mathrm{Nd}^{57+}$ (orange or gray line) and ${ }^{150} \mathrm{Nd}^{57+}$ (black line) in the energy range of the $1 s^{2} 2 p_{1 / 2} 18 l_{j^{\prime}}$ resonance groups. The labels indicate the individual fine structure components $j^{\prime}$ of the $n=18$ Rydberg electron. nances was exploited to explore magnetic hyperfine effects in $\mathrm{Cu}$-like ${ }^{207} \mathrm{~Pb}^{53+}$ [23] and in Li-like ${ }^{45} \mathrm{Sc}^{18+}$ [24]. Lowlying resonance are well known for selected ions of intermediate charge state but are unlikely for heavy fewelectron ions. The Li-like ions under study in this Letter are ideal candidates for precision DR-IS experiments and provide an explicit and straightforward access to nuclear parameters. The $2 s$ electronic wave function and the nucleus possess large mutual overlap leading to strong nuclear size contributions. In any element, DR resonances with excitations $2 s \rightarrow 2 p_{1 / 2}$ and in some cases additional resonances belonging to $2 s-2 p_{3 / 2}$ transitions can be found in the favorable energy range below a few $10 \mathrm{eV}$.

Detailed descriptions of the recombination setup at the electron cooler of the ESR are given elsewhere [10,22,25]. Here, we concentrate on the issues specific for the IS experiment with $\mathrm{Li}$-like ${ }^{A} \mathrm{Nd}^{57+}$ : Momentum selected ${ }^{A} \mathrm{Nd}^{57+}$ ions with $A=142$ or $A=150$ were injected into the storage ring ESR and stored at an energy of $56.3 \mathrm{MeV} / \mathrm{u}$. In order to minimize lateral beam size and energy spread electron cooling was applied. For both isotopes an identical space-charge corrected cooling voltage of $U_{C}=30899 \mathrm{~V}$ was chosen, equivalent to an ion velocity $\beta_{i}=0.3328$ in units of the speed of light. In addition to its normal function as a beam cooling device, we operated the electron cooler as a target of free electrons. After a series of recombination measurements with the reference isotope $A=142$, the operation parameters of the accelerator and the storage ring were scaled with the known mass ratio to the new isotope $A=150$. Special care was taken to ensure the same alignment of the ion beam with respect to the electron beam. Nonzero collision energies were introduced by applying a sequence of swiftly modulated voltage steps $U_{d}$ to a cylindrical drift tube located in the overlap region of the two beams. The relative collision energy $E_{\mathrm{cm}}$ in the center-of-mass system can then be easily inferred

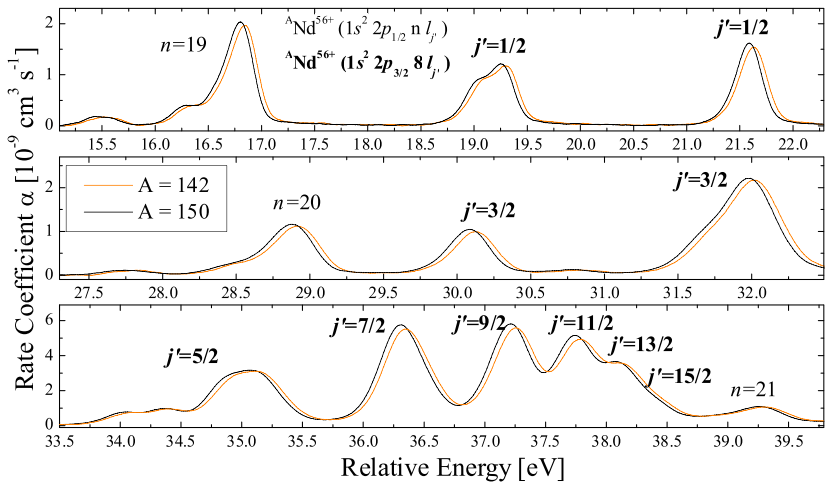

FIG. 2 (color online). Dielectronic recombination of $A=142$ (orange or gray) and $A=150$ (black) Li-like ${ }^{A} \mathrm{Nd}^{57+}$. The labels $n$ indicate the principal quantum number of the Rydberg electron of the $1 s^{2} 2 p_{1 / 2} n l_{j^{\prime}}$ configurations and the bold labels $j^{\prime}$ denote the fine structure components of the $1 s^{2} 2 p_{3 / 2} 8 l_{j^{\prime}}$ resonances. 
from the Lorentz factors $\gamma_{i}-1=e U_{C} /\left(m_{0, e} c^{2}\right)$ of the ion and $\gamma_{e}-1=e\left(U_{C}+U_{d}\right) /\left(m_{0, e} c^{2}\right)$ of the electrons. Here, $m_{0, e}$ is the electron mass. With $-2.5 \mathrm{kV} \leq U_{d} \leq$ $+2.5 \mathrm{kV}$ used in the present experiment, collision energies of $0<E_{\mathrm{cm}} \lesssim 50 \mathrm{eV}$ were covered. Recombined ions were separated from the primary beam in the next ESR bending magnet and were registered by a multiwire proportional chamber. A typical voltage sequence contained measurements at $\sim 4000$ different voltages of $33 \mathrm{~ms}$ duration each. Intermediate cooling steps of the same duration guaranteed a constant ion energy and beam quality. A constant electron current $I_{\mathrm{el}}=100 \mathrm{~mA}$ was applied, corresponding to a density $n_{e}=3.1 \times 10^{6} \mathrm{~cm}^{-3}$ at cooling. Comparable numbers of ions of typically about $N_{i}=2 \times 10^{7}$ for both isotopes were available in the ring. Recombination rate coefficients $\alpha$ were derived by normalizing the count rate using the primary beam intensities [22].

Isotope shift data were taken for three different energy ranges, $0-3.8 \mathrm{eV}, 12-24 \mathrm{eV}$, and $24-42 \mathrm{eV}$ with nominal step widths of $1 \mathrm{meV}, 3 \mathrm{meV}$, and $4 \mathrm{meV}$, respectively. With these settings, resonance groups ${ }^{A} \mathrm{Nd}^{56+}\left(1 s^{2} 2 p_{1 / 2} n l_{j^{\prime}}\right)^{* *}$ with $n=18 \ldots 21$ and ${ }^{A} \mathrm{Nd}^{56+}\left(1 s^{2} 2 p_{3 / 2} 8 l_{j^{\prime}}\right)^{* *}$ were covered. The shift of resonance positions for the two isotopes is immediately evident from the spectra in Figs. 1 and 2 and is clearly visible over the full energy range. For the extraction of the IS values, maxima, minima, and inflection points of the resonance spectra were used. By evaluating the shift of these characteristic points, errors due to normalization and background subtraction are minimized, and an analysis is achieved that is independent of supplementary input on the DR process and independent of the experimental response function. First and second derivative spectra (Fig. 3) as well as smoothed versions of the original spectrum were obtained from local fits of thirdorder polynomials for every point $i$ of the spectrum taking into account $m$ adjacent values on both sides of $i$. Full

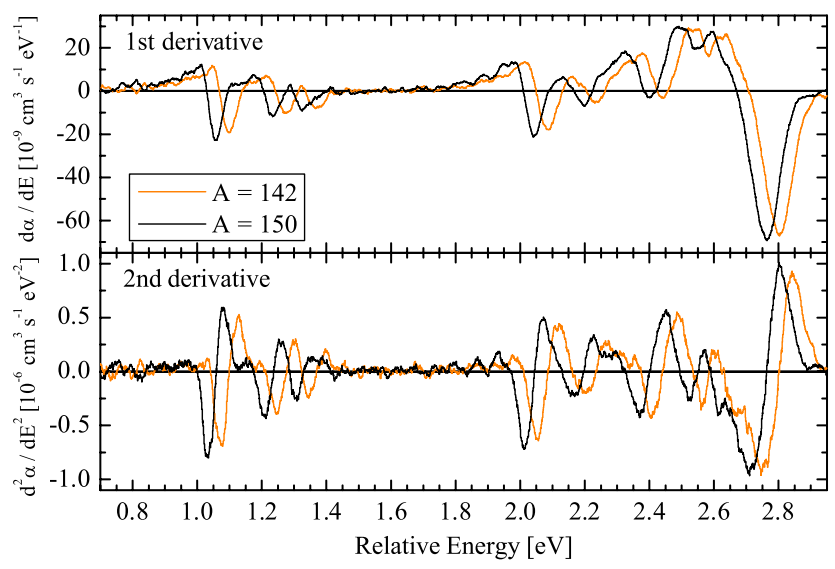

FIG. 3 (color online). First (upper panel) and second (lower panel) derivatives of the DR resonance spectra of the ${ }^{A} \mathrm{Nd}^{56+} 1 s^{2} 2 p_{1 / 2} 18 l_{j^{\prime}}$ group. The energy range is the same as in Fig. 1 . minimizations are performed accounting for a nonequidistant energy axis and for the error bars in both coordinates. The procedure is similar to the Savitzky and Golay smoothing algorithm [26] that retains fine details in the spectrum much better than averaging or rebinning [26]. For the analysis $m=35$ was used and no significant bias for $m=25$ or $m=45$ was found. For the IS determination only those roots of the derivatives were included that are significantly above the noise level. For the lowest energy range 7 independent IS data sets were obtained, 3 for the middle one and 1 for the energy range $24-42 \mathrm{eV}$ yielding in total 154 characteristic values for the $2 s-2 p_{1 / 2}$ IS and 45 for the $2 s-2 p_{3 / 2}$ IS. The average ISs are $\delta E^{142150}\left(2 s-2 p_{1 / 2}\right)=40.2(3)(6) \mathrm{meV}$ and a slightly higher value of $\delta E^{142150}\left(2 s-2 p_{3 / 2}\right)=42.3(12) \times$ (20) $\mathrm{meV}$. Here, first and second parentheses denote statistical and systematic errors. Although statistically not significant, the experimentally observed small difference in the ISs is expected, since in the relativistic case the $p_{1 / 2}$ electron has a finite overlap with the nucleus. The main sources of systematic errors stem from "slope" uncertainties, i.e., due to still remaining imperfections in background subtraction and normalization and from the finite digitalization of the energy steps. As a consequence of the merged-beams geometry, only minor contributions of below $0.2 \mathrm{meV}$ for the $2 p_{1 / 2}$ value and of $\sim 0.6 \mathrm{meV}$ for the $2 p_{3 / 2}$ IS arise from a potential beam misalignment or voltage calibration uncertainties of $U_{C}$ and $U_{d}$.

The IS values were evaluated within two different fully relativistic atomic structure methods, namely, the multiconfiguration Dirac-Fock (MCDF) and the configuration interaction Dirac-Fock-Sturmian methods (see [21], and references therein). The nucleus was modeled with a twoparameter Fermi distribution $\rho(r)=N(1+\exp [(r-$ $c) / a])^{-1}$, where $c$ is the half-density radius, $N$ is a normalization constant, and $t=4 a \ln 3$ the skin thickness [15]. IS measurements are basically insensitive to details of the charge distribution as long as the same root-mean-square (rms) radius is reproduced (e.g., [4,15,27]). We employed $t=2.3 \mathrm{fm}$ for both isotopes in accordance to the established value used for heavy ions [13-15,20,27]. The semimagic isotope ${ }^{142} \mathrm{Nd}$ (82 neutrons) served as a reference using the rms radius of $\left\langle r^{2}\right\rangle^{1 / 2}=4.9118 \mathrm{fm}$ from the most recent compilation of Angeli [16]. The radii values of Angeli were obtained from a combined analysis of comprehensive experimental input data from the four classical methods. The neutron-rich isotope ${ }^{150} \mathrm{Nd}(N=90)$ is strongly deformed yielding a pronounced increase of the charge radius beyond the liquid drop model [4].

For the $2 s-2 p$ transitions of ${ }^{A} \mathrm{Nd}^{57+}$ the total mass shift $A=142-A=150$ is as small as $\sim 4 \%$ of the IS and sums up to $-1.63 \mathrm{meV}$ for the $2 s \rightarrow 2 p_{1 / 2}$ excitation and to $-1.80 \mathrm{meV}$ for $2 s \rightarrow 2 p_{3 / 2}$, respectively. These values for the mass shift were obtained taking into account relativistic and QED recoil contributions [21,28]: For the 
$2 s-2 p_{1 / 2}\left(2 s-2 p_{3 / 2}\right)$ transition the mass shift comprises $-2.44 \mathrm{meV}(-2.53 \mathrm{meV})$ from averaging of the nonrelativistic recoil operator with relativistic multiconfiguration wave functions, $1.14 \mathrm{meV}(1.03 \mathrm{meV})$ from the relativistic recoil operator, and $-0.33 \mathrm{meV}(-0.30 \mathrm{meV})$ from the QED recoil effect. For the determination of $\delta\left\langle r^{2}\right\rangle$, influences of nuclear size (NS) variations on Coulomb electron correlation, on the Breit interaction as well as on QED contributions were taken into account. Similar to OIS [4], to a good approximation the IS depends linearly on $\delta\left\langle r^{2}\right\rangle$, yielding a slope of $-31.1 \mathrm{meV} / \mathrm{fm}^{2}$ for the $2 p_{1 / 2}$ and $-32.3 \mathrm{meV} / \mathrm{fm}^{2}$ for the $2 p_{3 / 2}$ transition, respectively. A further small correction that is often neglected [4] arises from nuclear polarization (NP). It is sizable for deformed nuclei with high nuclear transition probabilities $B(\tau L)$ such as ${ }^{150} \mathrm{Nd}$. Using the analytic formulas of [29] we estimate for the dominant contribution from the first excited $2^{+}$state at $130.21 \mathrm{keV}\left(B(E 2 \uparrow)=2.760 e^{2} b^{2}\right.$, [30]) a value of $-0.3 \mathrm{meV}$. Finally, from the NP corrected value of $\delta E^{142150}\left(2 s-2 p_{1 / 2}\right)=40.5(3)(6) \mathrm{meV}$, a change of ${ }^{142} 150 \delta\left\langle r^{2}\right\rangle=-1.36(1)(3) \mathrm{fm}^{2}$ is derived. Consistently, we obtain ${ }^{142} 150 \delta\left\langle r^{2}\right\rangle=-1.38(4)(7) \mathrm{fm}^{2}$ from the $2 p_{3 / 2}$ resonances but with significantly larger error bars. The systematic errors include additional theoretical uncertainties of $0.01 \mathrm{fm}^{2}$, predominantly from NP. We have added the full NP contribution to the error balance, since NP still lacks experimental verification. Our result of $-1.36(1) \times$ (3) $\mathrm{fm}^{2}$ is somewhat larger than the average experimental value of ${ }^{142}{ }^{150} \delta\left\langle r^{2}\right\rangle=-1.291(6) \mathrm{fm}^{2}$ from the combined analysis in Angeli [16]. However, our values obtained with this alternative method are in good agreement with the upper end of the individual data that build the basis of this evaluation (see discussion on the first page) and support a larger increase of the radius from $A=142$ to $A=$ 150.

In this Letter a novel method for nuclear size studies was developed and its general feasibility was demonstrated for heavy three-electron isotopes. These simple systems allow for a reliable analysis of the IS data within state-of-the-art structure calculations taking into account relativistic and QED contributions. ISs could be measured with $<1 \mathrm{meV}$ accuracy. For heavier ions a similar experimental precision can be retained. Because of the $Z^{5}$ to $Z^{6}$ scaling of nuclear size effects, for the heaviest ions ISs are of the order of $100 \mathrm{meV}$ [31] and hence the sensitivity of DR to the charge distribution is significantly enhanced. In contrast to OIS, isoelectronic studies of different elements are possible in order to disentangle atomic and nuclear contributions. High experimental resolution and large resonant atomic cross sections enable future DR-IS experiments with unstable isotopes or long-lived nuclear isomers. At GSI, such exotic highly charged ions can be synthesized in the frag- ment separator FRS [32] and subsequently investigated in the ESR. Thus, long chains with constant neutron number (isotone shifts) become accessible as well. At the upcoming Facility for Antiproton and Ion Research (FAIR) [33] high production yields of radioactive ions and a dedicated electron target at the New Experimental Storage Ring (NESR), will furthermore increase accuracy, versatility, and expand the scope of application.

*c.brandau@gsi.de

[1] N.D. Cook, Models of the Atomic Nucleus (Springer, Berlin, Heidelberg, New York, 2006).

[2] M. Bender et al., Rev. Mod. Phys. 75, 121 (2003).

[3] A. K. Jain et al., Rev. Mod. Phys. 62, 393 (1990).

[4] E. W. Otten, in Treatise on Heavy-Ion Science (Plenum, New York, 1989), Vol. 8, p. 517.

[5] D. Andrae, Phys. Rep. 336, 413 (2000).

[6] H. Hühnermann, Phys. Scr. T47, 70 (1993).

[7] M. Maul et al., Phys. Rev. A 53, 3915 (1996).

[8] E. N. Fortson et al., Phys. Rev. Lett. 65, 2857 (1990); 66, 677(E) (1991).

[9] V. A. Dzuba et al., Phys. Rev. A 72, 022503 (2005).

[10] C. Brandau et al., Phys. Rev. Lett. 91, 073202 (2003).

[11] P. Beiersdorfer et al., Phys. Rev. Lett. 95, 233003 (2005).

[12] A. Gumberidze et al., Phys. Rev. Lett. 94, 223001 (2005).

[13] P. J. Mohr et al., Phys. Rep. 293, 227 (1998).

[14] T. Beier et al., Phys. Rev. A 58, 954 (1998).

[15] G. Fricke et al., At. Data Nucl. Data Tables 60, 177 (1995).

[16] I. Angeli, At. Data Nucl. Data Tables 87, 185 (2004); Nuclear Data Services of the IAEA, INDC(HUN)-033, September 1999, http://www-nds.iaea.org/indc_sel.html.

[17] D. W. Madsen et al., Nucl. Phys. A168, 97 (1971).

[18] R. Maas et al., Phys. Lett. B 48, 212 (1974).

[19] B. Franzke, Nucl. Instrum. Methods Phys. Res., Sect. B 24/25, 18 (1987).

[20] S. R. Elliott et al., Phys. Rev. Lett. 76, 1031 (1996); 77, 4278(E) (1996).

[21] R. Soria Orts et al., Phys. Rev. Lett. 97, 103002 (2006).

[22] C. Brandau et al., Phys. Rev. Lett. 89, 053201 (2002).

[23] R. Schuch et al., Phys. Rev. Lett. 95, 183003 (2005).

[24] M. Lestinsky et al., Phys. Rev. Lett. 100, 033001 (2008).

[25] A. Hoffknecht et al., Phys. Rev. A 63, 012702 (2000).

[26] A. Savitzky et al., Anal. Chem. 36, 1627 (1964).

[27] V. M. Shabaev, J. Phys. B 26, 1103 (1993).

[28] A. N. Artemyev et al., Phys. Rev. A 52, 1884 (1995).

[29] A. V. Nefiodov et al., Phys. Lett. A 222, 227 (1996).

[30] S. Raman et al., At. Data Nucl. Data Tables 78, 1 (2001).

[31] C. Brandau et al., Nucl. Instrum. Methods Phys. Res., Sect. B 205, 66 (2003).

[32] H. Geissel et al., Nucl. Instrum. Methods Phys. Res., Sect. B 70, 286 (1992).

[33] Cf., http://www.fair-center.org/ (2007). 